\title{
THE APPLICATION OF ANALYTIC HIERARCHY PROCESS TO SUPPORT BUSINESS SELECTION FOR LAZ SIDOGIRI
}

\author{
Achmad Fitro \\ Computer Technology \\ NSC Polytechnic, Surabaya, East Java, Indonesia
}

\begin{abstract}
In essence, zakat must be distributed among eight predetermined groups, namely: indigent, poor, amil, ghorim, slave, ibn sabil, fisabilillah and converts. LAZ sidogiri is an Amil Zakat Institution located in Sidogiri Village which has the hope of changing zakat recipients (mustahik) into zakat givers (Muzakki) by providing community assistance in the form of capital for entrepreneurship. The provision of assistance by LAZ Sidogiri has been carried out for several periods. However, the assistance that has been carried out is still far from expectations because many recipients are still blind to knowledge of the types of businesses. The wealth of knowledge about types of business is considered to be the main factor, by having this knowledge the recipient can anticipate mistakes in determining the type of business such as the inaccuracy of the business towards the location, intense competition with the same business actors and unfair price competition. The Analytic hierrarchy process method is a very popular method of making decisions by giving priority values to each variable. That way, the decision support system using the AHP method can help recipients of assistance to meet the expectations given by LAZ Sidogiri
\end{abstract}

Keywords - Decision support, Analytic Hierarchy Process, Zakat, Enterpreuner, UMKM

\section{INTRODUCTION}

In essence, zakat is distributed or distributed to 8 (eight) predetermined groups, namely: needy, poor, amil, ghorim, slave, ibn sabil, fisabilillah and converts [1]. Zakat is one of the pillars of Islam and must be carried out by some individuals who have a lot of assets (according to the nisab) as a process of cleaning and purifying for them [2], often zakat is given to mustahik to fulfill all consumptive life needs such as: daily shopping, loans and education [3]. However, the giving of zakat which is consumptive in nature cannot fully guarantee the welfare of the mustahik so that zakat is prioritized on a productive nature such as the provision of capital and employment [4]. that way the management of zakat must really be managed properly in accordance with Islamic law so as not to be misdirected like LAZ Sidogiri.

\author{
Heru Prasetyo \\ Computer Technology \\ NSC Polytechnic, Surabaya, East Java, Indonesia
}

LAZ Sidogiri is an Amil Zakat Institution that has distributed productive zakat in the form of training, business capital [5] and has hopes of changing Mustahik (recipient) to Muzakki (giver) [6]. The hope of zakat distribution that has been carried out by LAZ Sidogiri is still far from ideal. Many zakat recipients who have been selected are still confused in determining what line of business to run, so that many Mustahik run an inaccurate business sector which can cause the business to stop such as: selection of business by location, intense competition in the same field, range of promotion and product manufacture. LAZ Sidogiri hopes to be able to assist in making decisions regarding business sector selection by taking into account several criteria.

The multi-criteria decision-making method is very fast developing among researchers in supporting policies in a company such as the AHP method to create an idea related to corporate social responsibility (CSR) related to quality, strategy, sustainability and image of an airline company [7], TOPSIS selection of building materials [8], FAHP to determine mineral water development points [9] and AHP Fuzzy and VIKOR in development in the investment sector [10]. That way, the use of the multi-criteria method can solve the problems faced by LAZ Sidogiri, one of which is the AHP method.

\section{PROPOSED ALGORITHM}

\section{A. Analytic Hierarrchy Process (AHP}

AHP has a strong potential in structuring decision problems in the form of a hierarchical structure. In general, the form of a hierarchical structure takes the form of a tree where the roots represent the overall goal and the nodes descending from the goals represent the criteria. The complexity of the decision problem controls the number of levels of the main criterion and alternative criteria, the last level of the structure is stored for the selection set [11]. AHP uses pairwise comparisons at each node of the structure and allows consistency for cross-checking between different paired comparisons using a ratio scale [12]. Pairwise comparisons are a mainstay in reducing the impact of subjective viewpoints associated with direct weighting [13]. The AHP method makes it possible to evaluate the quantitative and qualitative criteria 


\section{International Journal of Engineering Applied Sciences and Technology, 2020 \\ Vol. 5, Issue 6, ISSN No. 2455-2143, Pages 64-71 \\ Published Online October 2020 in IJEAST (http://www.ijeast.com)}

of alternatives on the same preference scale at each level where verbal comparisons must be converted into numerical values [14]. Derivation of priority in the ahp method requires calculating the maximum Eigenvalues, Consistency Index (CI), Consistency ratio, and normal values for each criterion / alternative and obtaining satisfactory results, otherwise the procedure will be repeated until these values are well within the desired range. Therefore, researchers can conclude the AHP method has the following steps:

1. Determination of Criteria and Alternative Elements In applying the AHP method, we must first define the hierarchical structure obtained from the criteria and alternative elements as shown in Fig 1:

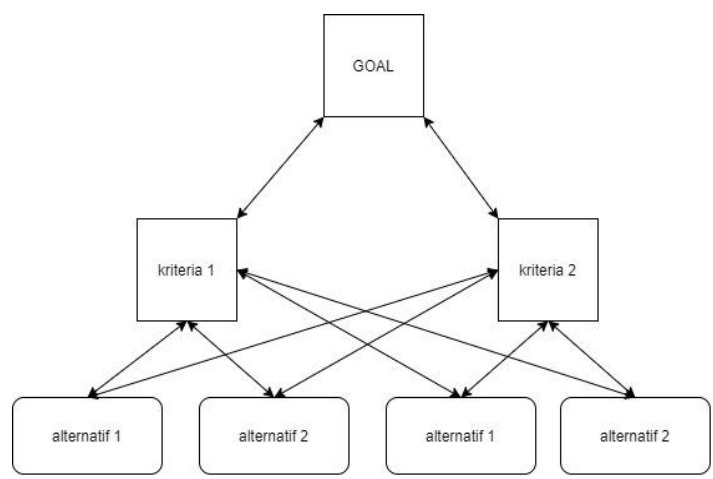

Fig 1. Hierarchical structure

2. Create pairwise matrix

After each element forms a hierarchical structure, the next step is to build a paired matrix against each criterion as many as the number of criteria that have been determined. The paired matrix can be explained in Table 1

Table - 1 Paired Matrix

\begin{tabular}{|c|c|c|c|c|}
\hline & Criteria 1 & Criteria $_{2}$ & ......... & Criteria $_{n}$ \\
\hline Criteria $_{1}$ & & & & \\
\hline Criteria $_{2}$ & & & & \\
\hline$\ldots$. & & & & \\
\hline Criteria n & & & & \\
\hline
\end{tabular}

\section{Weighted Elements}

Each level of the hierarchy must be assigned a value / weight according to the provisions in Table 2:

Table - 2 Wieghting Scale [15]

\begin{tabular}{|r|l|}
\hline 1 & Just as important \\
\hline 2 & The same is a little more important \\
\hline 3 & A little bit more important \\
\hline 4 & $\begin{array}{l}\text { A little more until it is clearly more } \\
\text { important }\end{array}$ \\
\hline 5 & Obviously more important \\
\hline 6 & Clear to very clear is more important \\
\hline
\end{tabular}

\begin{tabular}{|r|l|}
\hline 7 & Very clearly more important \\
\hline 8 & $\begin{array}{l}\text { So clear that it is absolutely more } \\
\text { important }\end{array}$ \\
\hline 9 & Absolutes are more important \\
\hline
\end{tabular}

4. Calcating Consistency Priority Value

The consistency priority value will be replaced by an $\mathrm{X}$ symbol in the paired matrix in Table 1 with the provision of contributions based on the weighted value of the elements in Table 2 which will be explained in Table 3 .

Table -3 Calculation of consistency priority value

\begin{tabular}{|c|c|c|c|c|}
\hline & Criteria 1 & Criteria $_{2}$ & $\ldots$ & Criteria $n$ \\
\hline Criteria $_{1}$ & 1 & $\mathrm{X}_{2}$ & & $\mathrm{X}_{\mathrm{n}}$ \\
\hline Criteria $_{2}$ & $1 / X_{1}$ & 1 & & \\
\hline$:$ & & & 1 & \\
\hline Criteria $_{n}$ & $1 / X_{n}$ & & & 1 \\
\hline Jumlah & $\sum$ Criteria $_{1}$ & $\sum$ Criteria $_{2}$ & $\sum$ Criteria $_{3}$ & $\sum$ Criteria $_{1}$ \\
\hline
\end{tabular}

After obtaining the number per column, the contents of the matrix are changed into the Criteria Value matrix by dividing from the results of the sum of each Criteria in Table 3

5. Calculating the Eigen Vector

After getting the total value per line, we can calculate the Eigen Vector as shown in Table 4.

Table -4 Eigen vector calculation

\begin{tabular}{|c|c|c|c|}
\hline & Total & Priority & $\begin{array}{c}\text { Solution (Total }+ \\
\text { Priority) }\end{array}$ \\
\hline Criteria $_{1}$ & $\sum$ Criteria $_{1}$ & $\sum$ Criteria $_{1} / \mathrm{n}$ & $\sum$ Criteria $_{1}+\sum$ Criteria $_{1} / \mathrm{n}$ \\
\hline Criteria $_{2}$ & $\sum$ Criteria $_{2}$ & $\sum$ Criteria $_{2} / \mathrm{n}$ & $\sum$ Criteria $_{2}+\sum$ Criteria $_{2} / \mathrm{n}$ \\
\hline$:$ & $:$ & $:$ & $:$ \\
\hline Criteria $_{n}$ & $\sum$ Criteria $_{n}$ & $\sum$ Criteria $_{n} / \mathrm{n}$ & $\sum$ Criteria $_{n}+\sum$ Criteria $_{n} / \mathrm{n}$ \\
\hline & & Total & $\sum$ Solusi \\
\hline
\end{tabular}

Information :

Eigen Maksimum $(\lambda \mathrm{mak})=\sum$ Solusi $/ \mathrm{n}$

Consistency Indexs $(\mathrm{CI})=(\lambda \mathrm{mak}-\mathrm{n}) /(\mathrm{n}-1)$

Where $\mathrm{n}$ is the sum of the Criteria matrix

6. Random Index (RI)

Random Index (RI) is a constant value used for Consistency Random calculations, the following values will be described in Table 5 .

Table - 5 Random Index (RI)

\begin{tabular}{|c|c|}
\hline $\mathrm{n}$ & $\mathrm{RI}$ \\
\hline 1 & 0,0 \\
\hline
\end{tabular}




\begin{tabular}{|c|c|}
2 & 0,0 \\
\hline 3 & 0,58 \\
\hline 4 & 0,9 \\
\hline 5 & 1,12 \\
\hline 6 & 1,24 \\
\hline 7 & 1,32 \\
\hline 8 & 1,41 \\
\hline 9 & 1,45 \\
\hline 10 & 1,49 \\
\hline 11 & 1,51 \\
\hline 12 & 1,48 \\
\hline 13 & 1,56 \\
\hline 14 & 1,57 \\
\hline 15 & 1,59 \\
\hline
\end{tabular}

7. Ratio Consistency

Consistency Ratio (CR) is the consistency of opinion on decisions taken. The formula for getting $\mathrm{CR}$ is in equation 3 .

$$
\mathrm{CR}=\mathrm{CI} / \mathrm{RI}
$$

If the $\mathrm{CR}$ result is less than $10 \%$, then the decision can be tolerated or accepted.

\section{EXPERIMENT AND RESULT}

The system created will be divided into two parts (source code), namely the web-based admin role and the android mobile application (apk) based user. The implementation of the AHP method in this system has been determined in a hierarchical structure which will be described in Fig 2 according to the problems faced with basic aspects of business planning [16-17].

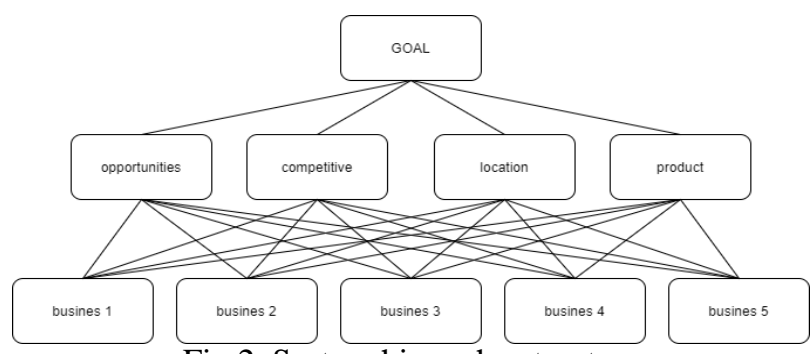

Fig 2. System hierarchy structure

A. Role administrator

The admin on this system will get a login page before entering the system as shown in Fig 3 below.

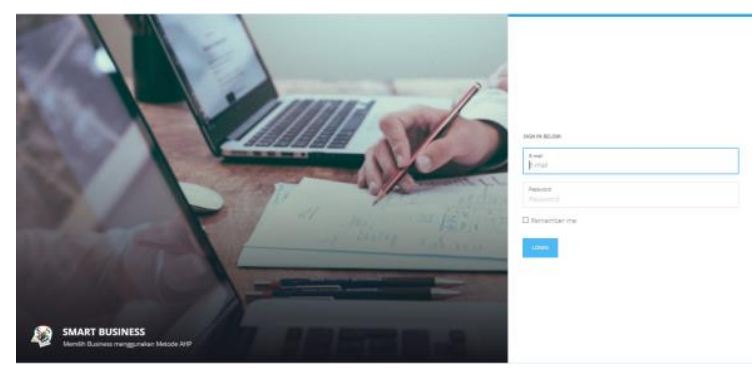

Fig 3. Page login

After successfully entering the system, the admin will get menus in managing the system such as the ahp module, entrepreneurial module, settings, and tools which will be explained in Fig 4.

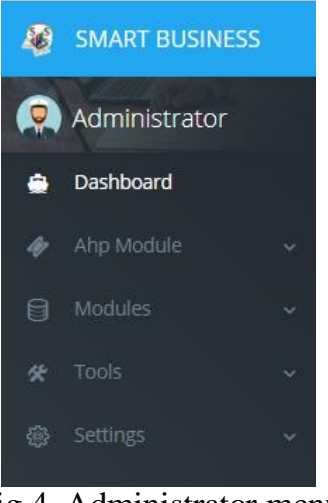

Fig 4. Administrator menu

On the Ahp module menu, the administrator must fill in a weighting scale, random index (RI), Criteria and perform pairwise comparison calculations so that it will produce a value matrix for each Criteria as explained in Fig 5 which will affect the results of user analysis.

in this application, the administrator is tasked with calculating the matrix between one alternative and another. The administrator is trusted to fill in because the administrator is a representative part of the site surveyor. The following is the pairwise comparison calculation for the alternatives which will be explained in the table 6-13. 

International Journal of Engineering Applied Sciences and Technology, 2020

Vol. 5, Issue 6, ISSN No. 2455-2143, Pages 64-71

Published Online October 2020 in IJEAST (http://www.ijeast.com)

Table - 6 Matrix pairwise opportunities

\begin{tabular}{|l|c|c|c|c|c|}
\hline Criteria & $\begin{array}{c}\text { Usaha Risoles / Sosis } \\
\text { Solo }\end{array}$ & Jamu Kunyit Asam & $\begin{array}{c}\text { Ekspedisi } \\
\text { lionparcel }\end{array}$ & Ekspedisi Wahana & Pakaian Anak \\
\hline Usaha Risoles / Sosis Solo & 1 & 0.5 & 5 & 4 & 0.5 \\
\hline Jamu Kunyit Asam & 2 & 1 & 6 & 5 & 1 \\
\hline Ekspedisi lionparcel & 0.2 & 0.16667 & 1 & 0.5 & 0.16667 \\
\hline Ekspedisi Wahana & 0.25 & 0.2 & 2 & 5 & 1 \\
\hline Pakaian Anak & 2 & 1 & 6 & 5 & 1 \\
\hline SUM & 5.45 & 2.86667 & 20 & & 2.2 \\
\hline
\end{tabular}

Table - 7 Eigen vector calculation opportunities

\begin{tabular}{|c|c|c|c|c|c|c|c|}
\hline Kriteria & $\begin{array}{c}\text { Usaha Risoles / } \\
\text { Sosis Solo }\end{array}$ & Jamu Kunyit Asam & $\begin{array}{l}\text { Ekspedisi } \\
\text { lionparcel }\end{array}$ & $\begin{array}{c}\text { Ekspedisi } \\
\text { Wahana }\end{array}$ & Pakaian Anak & Jumlah & $\begin{array}{r}\text { Priority } \\
\text { Vector }\end{array}$ \\
\hline $\begin{array}{l}\text { Usaha Risoles / Sosis } \\
\text { Solo }\end{array}$ & 0.18349 & 0.17442 & 0.25 & 0.25806 & 0.17442 & 1.04039 & 0.20808 \\
\hline Jamu Kunyit Asam & 0.36697 & 0.34884 & 0.3 & 0.32258 & 0.34884 & 1.68723 & 0.33745 \\
\hline Ekspedisi lionparcel & 0.0367 & 0.05814 & 0.05 & 0.03226 & 0.05814 & 0.23523 & 0.04705 \\
\hline Ekspedisi Wahana & 0.04587 & 0.06977 & 0.1 & 0.06452 & 0.06977 & 0.34992 & 0.06998 \\
\hline Pakaian Anak & 0.36697 & 0.34884 & 0.3 & 0.32258 & 0.34884 & 1.68723 & 0.33745 \\
\hline \multicolumn{7}{|c|}{ Principe Eigen Vector $(\lambda$ maks $)$} & 5.09441 \\
\hline \multicolumn{7}{|c|}{ Consistency Index } & 0.0236 \\
\hline \multicolumn{7}{|c|}{ Consistency Ratio } & $2.11 \%$ \\
\hline
\end{tabular}

Table - 8 Matrix pairwise competitive

\begin{tabular}{|l|c|c|c|c|c|}
\hline Criteria & $\begin{array}{c}\text { Usaha Risoles / Sosis } \\
\text { Solo }\end{array}$ & Jamu Kunyit Asam & $\begin{array}{c}\text { Ekspedisi } \\
\text { lionparcel }\end{array}$ & Ekspedisi Wahana & Pakaian Anak \\
\hline Usaha Risoles / Sosis Solo & 1 & 0.5 & 3 & 5 & \\
\hline Jamu Kunyit Asam & 2 & 1 & 4 & 6 & 6 \\
\hline Ekspedisi lionparcel & 0.33333 & 0.25 & 1 & 3 & 1 \\
\hline Ekspedisi Wahana & 0.2 & 0.16667 & 0.33333 & 0.33 & 1 \\
\hline Pakaian Anak & 0.2 & 0.16667 & 8.66667 & 16 & 1 \\
\hline Jumlah & 3.73333 & 2.08333 & & & 16 \\
\hline
\end{tabular}

Table -9 Eigen vector calculation competitve

\begin{tabular}{|l|c|c|c|c|c|c|c|}
\hline Criteria & $\begin{array}{c}\text { Usaha Risoles / } \\
\text { Sosis Solo }\end{array}$ & Jamu Kunyit Asam & $\begin{array}{c}\text { Ekspedisi } \\
\text { lionparcel }\end{array}$ & $\begin{array}{c}\text { Ekspedisi } \\
\text { Wahana }\end{array}$ & Pakaian Anak & Jumlah & $\begin{array}{c}\text { Priority } \\
\text { Vector }\end{array}$ \\
\hline $\begin{array}{l}\text { Usaha Risoles / Sosis } \\
\text { Solo }\end{array}$ & 0.26786 & 0.24 & 0.34615 & 0.3125 & 0.3125 & 1.47901 & 0.2958 \\
\hline Jamu Kunyit Asam & 0.53571 & 0.48 & 0.46154 & 0.375 & 0.375 & 2.22725 & 0.44545 \\
\hline Ekspedisi lionparcel & 0.08929 & 0.12 & 0.11538 & 0.1875 & 0.1875 & 0.69967 & 0.13993 \\
\hline Ekspedisi Wahana & 0.05357 & 0.08 & 0.03846 & 0.0625 & 0.0625 & 0.29703 & 0.05941 \\
\hline Pakaian Anak & 0.05357 & 0.08 & 0.03846 & 0.0625 & 0.0625 & 0.29703 & 0.05941 \\
\hline \multicolumn{7}{|c|}{ Principe Eigen Vector $(\lambda$ maks) } \\
\hline \multicolumn{7}{|c|}{ Consistency Index } \\
\hline \multicolumn{7}{|c|}{ Consistency Ratio } \\
\hline
\end{tabular}


International Journal of Engineering Applied Sciences and Technology, 2020

Vol. 5, Issue 6, ISSN No. 2455-2143, Pages 64-71

Published Online October 2020 in IJEAST (http://www.ijeast.com)

Table - 10 Matrix pairwise location

\begin{tabular}{|l|c|c|c|c|c|}
\hline Criteria & $\begin{array}{c}\text { Usaha Risoles / Sosis } \\
\text { Solo }\end{array}$ & Jamu Kunyit Asam & $\begin{array}{c}\text { Ekspedisi } \\
\text { lionparcel }\end{array}$ & Ekspedisi Wahana & Pakaian Anak \\
\hline Usaha Risoles / Sosis Solo & 1 & 1 & 3 & 3 & 1 \\
\hline Jamu Kunyit Asam & 1 & 1 & 3 & 3 & 1 \\
\hline Ekspedisi lionparcel & 0.33333 & 0.33333 & 1 & 1 & 0.33333 \\
\hline Ekspedisi Wahana & 0.33333 & 0.33333 & 1 & 1 & 0.33333 \\
\hline Pakaian Anak & 1 & 1 & 3 & 3 & 1 \\
\hline SUM & 3.66667 & 3.66667 & 11 & 3.66667 \\
\hline
\end{tabular}

Table - 11 Eigen vector calculation location

\begin{tabular}{|l|c|c|c|c|c|c|c|}
\hline Criteria & $\begin{array}{c}\text { Usaha Risoles / } \\
\text { Sosis Solo }\end{array}$ & Jamu Kunyit Asam & $\begin{array}{c}\text { Ekspedisi } \\
\text { lionparcel }\end{array}$ & $\begin{array}{c}\text { Ekspedisi } \\
\text { Wahana }\end{array}$ & Pakaian Anak & Jumlah & $\begin{array}{c}\text { Priority } \\
\text { Vector }\end{array}$ \\
\hline $\begin{array}{l}\text { Usaha Risoles / Sosis } \\
\text { Solo }\end{array}$ & 0.27273 & 0.27273 & 0.27273 & 0.27273 & 0.27273 & 1.36364 & 0.27273 \\
\hline Jamu Kunyit Asam & 0.27273 & 0.27273 & 0.27273 & 0.27273 & 0.27273 & 1.36364 & 0.27273 \\
\hline Ekspedisi lionparcel & 0.09091 & 0.09091 & 0.09091 & 0.09091 & 0.09091 & 0.45455 & 0.09091 \\
\hline Ekspedisi Wahana & 0.09091 & 0.09091 & 0.09091 & 0.09091 & 0.09091 & 0.45455 & 0.09091 \\
\hline Pakaian Anak & 0.27273 & 0.27273 & 0.27273 & 0.27273 & 0.27273 & 1.36364 & 0.27273 \\
\hline \multicolumn{7}{|c|}{ Principe Eigen Vector $(\lambda$ maks) } \\
\hline \multicolumn{7}{|c|}{ Consistency Index } \\
\hline \multicolumn{7}{|c|}{ Consistency Ratio } \\
\hline
\end{tabular}

Table - 12 Matrix pairwise product

\begin{tabular}{|l|c|c|c|c|c|}
\hline Criteria & $\begin{array}{c}\text { Usaha Risoles / Sosis } \\
\text { Solo }\end{array}$ & Jamu Kunyit Asam & $\begin{array}{c}\text { Ekspedisi } \\
\text { lionparcel }\end{array}$ & Ekspedisi Wahana & Pakaian Anak \\
\hline Usaha Risoles / Sosis Solo & 1 & 3 & 0.5 & 0.33333 & 1 \\
\hline Jamu Kunyit Asam & 0.33333 & 1 & 0.25 & 0.2 & 0.33333 \\
\hline Ekspedisi lionparcel & 2 & 4 & 1 & 0.5 & 1 \\
\hline Ekspedisi Wahana & 3 & 5 & 2 & 2 & 3 \\
\hline Pakaian Anak & 1 & 3 & 0.5 & 0.33333 & 1 \\
\hline SUM & 7.33333 & 16 & 4.25 & 2.36667 & 7.33333 \\
\hline
\end{tabular}

Table - 13 Eigen vector calculation product

\begin{tabular}{|c|c|c|c|c|c|c|c|}
\hline Criteria & $\begin{array}{c}\text { Usaha Risoles / } \\
\text { Sosis Solo }\end{array}$ & Jamu Kunyit Asam & $\begin{array}{l}\text { Ekspedisi } \\
\text { lionparcel }\end{array}$ & $\begin{array}{c}\text { Ekspedisi } \\
\text { Wahana }\end{array}$ & Pakaian Anak & Jumlah & $\begin{array}{l}\text { Priority } \\
\text { Vector }\end{array}$ \\
\hline $\begin{array}{l}\text { Usaha Risoles / Sosis } \\
\text { Solo }\end{array}$ & 0.13636 & 0.1875 & 0.11765 & 0.14085 & 0.13636 & 0.71872 & 0.14374 \\
\hline Jamu Kunyit Asam & 0.04545 & 0.0625 & 0.05882 & 0.08451 & 0.04545 & 0.29674 & 0.05935 \\
\hline Ekspedisi lionparcel & 0.27273 & 0.25 & 0.23529 & 0.21127 & 0.27273 & 1.24202 & 0.2484 \\
\hline Ekspedisi Wahana & 0.40909 & 0.3125 & 0.47059 & 0.42254 & 0.40909 & 2.02381 & 0.40476 \\
\hline Pakaian Anak & 0.13636 & 0.1875 & 0.11765 & 0.14085 & 0.13636 & 0.71872 & 0.14374 \\
\hline \multicolumn{7}{|c|}{ Principe Eigen Vector ( $\lambda$ maks) } & 5.07146 \\
\hline \multicolumn{7}{|c|}{ Consistency Index } & 0.01786 \\
\hline \multicolumn{7}{|c|}{ Consistency Ratio } & $1.60 \%$ \\
\hline
\end{tabular}




\section{International Journal of Engineering Applied Sciences and Technology, 2020 \\ Vol. 5, Issue 6, ISSN No. 2455-2143, Pages 64-71 \\ Published Online October 2020 in IJEAST (http://www.ijeast.com)}

\section{B. Role user}

The role user in this system is expected to be able to help mustahik who will run entrepreneurship. Before using the application, the user must first download the Playstore available on an Android phone, the name of the application on the Playstore will be shown in Fig. 5.
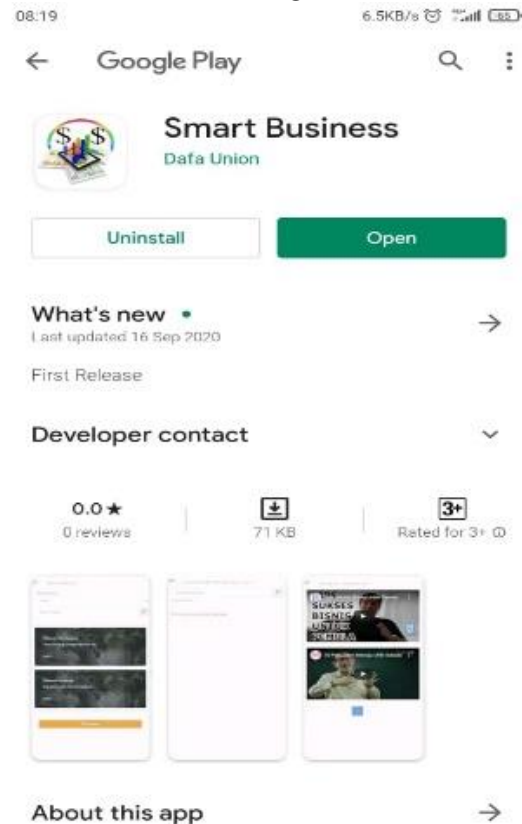

Fig 5. Smart business on the playstore

After downloading the application, the user will gain knowledge of the types of business fields, business news, video tutorials, marketplaces that sell business needs and business analysis if you are still confused about choosing a decision. Analysis using AHP for weight filling will be described in Table 14. However, before weighting the user will be asked to enter the nominal capital as a sorting business.

Table -14 Weighting scale

\begin{tabular}{|c|c|c|c|c|}
\hline \multicolumn{4}{|c|}{ Choice } & Nilai \\
\hline Product & $\sqrt{ }$ & Location & & 3 \\
\hline Product & & Competitive & $\sqrt{ }$ & 3 \\
\hline Product & & Opportunites & $\sqrt{ }$ & 2 \\
\hline Location & & Competititve & $\sqrt{ }$ & 5 \\
\hline Location & & Opportunites & $\sqrt{ }$ & 4 \\
\hline Competitive & $\sqrt{ }$ & Opportunites & & 2 \\
\hline
\end{tabular}

After filling in the comparison scale, the user will get the results of the pairwise comparison matrix such as Table 15 and the Criteria Value Matrix which will be explained in Table 16.

Table - 15 Matrix pairwise criteria

\begin{tabular}{|l|c|c|c|c|}
\hline Kriteria & Product & Location & Competitive & Opportunities \\
\hline Product & 1 & 3 & 0.33333 & 0.5 \\
\hline Location & 0.33333 & 1 & 0.2 & 0.25 \\
\hline Competitive & 3 & 5 & 1 & 2 \\
\hline Opportunities & 2 & 4 & 0.5 & 1 \\
\hline Jumlah & 6.33333 & 13 & 2.03333 & 3.75 \\
\hline
\end{tabular}

Table - 16 Eigen vector criteria

\begin{tabular}{|l|c|c|c|c|c|c|}
\hline Kriteria & Product & Location & Competitive & Opportunities & SUM & Priority Vector \\
\hline Product & 0.15789 & 0.23077 & 0.16393 & 0.13333 & 0.68593 & 0.17148 \\
\hline Location & 0.05263 & 0.07692 & 0.09836 & 0.06667 & 0.29458 & 0.07365 \\
\hline Competitive & 0.47368 & 0.38462 & 0.4918 & 0.53333 & 1.88344 & 0.47086 \\
\hline Opportunities & 0.31579 & 0.30769 & 0.2459 & 0.26667 & 1.13605 & 0.28401 \\
\hline \multicolumn{7}{|c|}{ Principe Eigen Vector $(\lambda$ maks) } \\
\hline \multicolumn{7}{|c|}{ Consistency Index } \\
\hline
\end{tabular}

After getting a Consistency Ratio below $10 \%$, namely $2.44 \%$, the user results can continue to get the calculation results as shown in Table 17.

Table - 17 Eigen vector criteria and alternative

\begin{tabular}{|c|c|c|c|c|c|c|}
\hline Overall Composite Height & $\begin{array}{c}\text { Priority Vector (rata- } \\
\text { rata) }\end{array}$ & $\begin{array}{c}\text { Usaha Risoles / Sosis } \\
\text { Solo } \\
\end{array}$ & $\begin{array}{c}\text { Jamu Kunyit } \\
\text { Asam } \\
\end{array}$ & $\begin{array}{l}\text { Ekspedisi } \\
\text { lionparcel } \\
\end{array}$ & $\begin{array}{c}\text { Ekspedisi } \\
\text { Wahana }\end{array}$ & $\begin{array}{c}\text { Pakaian } \\
\text { Anak }\end{array}$ \\
\hline Product & 0.17148 & 0.14374 & 0.05935 & 0.2484 & 0.40476 & 0.14374 \\
\hline Location & 0.07365 & 0.27273 & 0.27273 & 0.09091 & 0.09091 & 0.27273 \\
\hline Competitive & 0.47086 & 0.32865 & 0.4308 & 0.13516 & 0.0527 & 0.0527 \\
\hline Opportunities & 0.28401 & 0.20808 & 0.33745 & 0.04705 & 0.06998 & 0.33745 \\
\hline \multicolumn{2}{|c|}{ Total } & 0.25858 & 0.32895 & 0.12629 & 0.1208 & 0.16539 \\
\hline
\end{tabular}




\section{International Journal of Engineering Applied Sciences and Technology, 2020 \\ Vol. 5, Issue 6, ISSN No. 2455-2143, Pages 64-71 \\ Published Online October 2020 in IJEAST (http://www.ijeast.com)}

After calculating the eighen vector, the user will be able to see what type of business is most recommended by the system such as Table 18 with the tamarind herbal medicine business which has the first rank and the least recommended wahana expedition.

Table - 18 Ranking business recomended

\begin{tabular}{|c|c|c|}
\hline Rank & Alternatif & Nilai \\
\hline 1 & Jamu Kunyit Asam & 0.328945 \\
\hline 2 & Usaha Risoles / Sosis Solo & 0.258578 \\
\hline 3 & Pakaian Anak & 0.165388 \\
\hline 4 & Ekspedisi lionparcel & 0.126294 \\
\hline 5 & Ekspedisi Wahana & 0.120795 \\
\hline
\end{tabular}

\section{CONCLUSION}

From the results of the discussion, the authors can conclude several points, including: Mustahik will have a wealth of knowledge on the types of fields in entrepreneurship, Get tips and tricks in entrepreneurship, develop business innovation and can support a decision in determining the field of business using the method AHP on application.

\section{REFERENCE}

[1] Firdaningsih., Wahyudi, M.S., \& Hakim, R. (2019). Delapan Golongan Penerima Zakat Analisis Teks dan Konteks. Jurnal Ekonomi Syariah, 7(2), 316-342. https://doi.org/10.21043/equilibrium.v7i2.5843

[2] Hasanah, U. (2020). Optimalisasi Pengelolaan Zakat Produktif Sebagai Realisasi Revolusi Mental. Ijtimaiyya: Jurnal Pengembangan Masyarakat Islam, 13(1), 75-88.

[3] Abubakar, A.Y. (2014). Senif Penerima Zakat: Sebuah Upaya Untuk Reinterpretasi. Media Syariah, 16(1)

[4] Riyaldi, M.H. (2017). Kedudukan dan Prinsip Pembagian Zakat dalam Mengatasi Permasalah Kemiskinan (Analisis Pandangan Yusuf Qardhawi). Jurnal Perspektif Ekonomi Darussalam, 3(1).

[5] Hakim, A. (2015). Pengelolaan Zakat Pertanian Di Lazis $\mathrm{Nu}$ Kabupaten Kendal. Wahana Akademika, 2 (2)

[6] Ridwan, M., Andalsari, M., Setiani, R.I., \& Merliana, R. (2020). Pengelolaan Zakat Produktif Melalui Program Senyum Mandiri Dalam Pemberdayaan Ekonomi Mustahiq Di Rumah Zakat Cabang Cirebon. Jurnal Perbankan Syariah, Jurnal EcoBankers, 1(2),44 - 52.

[7] Karaman, A.S., \& Akman, E. (2017). Taking-off corporate social responsibility programs: An AHP application in airline industry. , Journal of Air Transport
Management,

68 http://dx.doi.org/10.1016/j.jairtraman.2017.06.012

[8] Singh, A.K., Avikal, S., Kumar, N.K.C., Kumar, M., \& Thakura, P. (2020). A fuzzy-AHP and M- TOPSIS based approach for selection of composite materials used in structural applications. Materials Today: Proceedings, 26(2),

$3119-3123$ https://doi.org/10.1016/j.matpr.2020.02.644

[9] Khasesi-Siuki, A., Keshavarz, A., \& Sharifan, H. (2020). Comparison of AHP and FAHP methods in determining suitable areas for drinking water harvesting in Birjand aquifer. Iran. Groundwater for Sustainable Development, 10 (100328). https://doi.org/10.1016/j.gsd.2019.100328

[10] Ozdemir, S., \& Sahin, G. (2018). Multi-criteria decisionmaking in the Location Selection for a Solar PV Power Plant using AHP. Measurement, 129, 218-226. https://doi.org/10.1016/j.measurement.2018.07.020

[11] Del Vasto-Terrientes, L., Valls, A., Slowinski, R., \& Zielniewicz, P. (2015). ELECTRE-III-H: An outrankingbased decision aiding method for hierarchically structured criteria. Expert Systems with Applications, 42(11), 49104926.

[12] Kainulainen, T., Leskinen, P., Korhonen, P., Haara, A., \& Hujala, T. (2009). A statistical approach to assessing interval scale preferences in discrete choice problems. Journal of the Operational Research Society, 60(2), 252258

[13] Dede, G., Kamalakis, T., \& Sphicopoulos, T. (2016). Theoretical estimation of the probability of weight rank reversal in pairwise comparisons. European Journal of Operational Research, 252(2), 587-600.

[14] Ishizaka, A., \& Labib, A. (2011). Review of the main developments in the analytic hierarchy process. Expert Systems with Applications, 38(11), 14336-14345.

[15] Saaty, T.L., \& Kirti, P. (2008). Group Decision Making: Drawing out and Reconciling Differences. RWS Publications: Pittsburgh Pennsylvania

[16] Tamjis, A.K., \& Fitro, A. 2018. Design of Web-Based Legislative Candidate Application. Journal of Information Systems and Applied Computer Science. 1 (1). 1-6

[17] Supriyanto. (2009). BUSINESS PLAN SEBAGAI LANGKAH AWAL MEMULAI USAHA. Jurnal Ekonomi \& Pendidikan, 6(1) 